\title{
Pfg NMR studies of lateral diffusion in oriented lipid bilayers
}

\author{
Greger Orädd and Göran Lindblom \\ Department of Chemistry: Biophysical Chemistry, Umeå University, SE-901 87 Umeå, Sweden
}

\begin{abstract}
The pfg-NMR diffusion technique is proposed to have an appreciable potential for future biophysical investigations in the field of membrane biology. Topics like transport of molecules both across and in the plane of the membrane can be successfully studied, as well as the formation of lipid domains and their intrinsic dynamics can be scrutinized. This short review will introduce the fundamental aspects of orientation dependent NMR interactions and the technique of macroscopically oriented bilayers for eliminating the unwanted effects of those interactions. The pfg-NMR technique will be briefly introduced and finally, some recent results illustrating the potential of the method are presented.
\end{abstract}

\section{Orientation dependent NMR interactions}

The NMR spectrum for a general spin system is determined by the spin Hamiltonian, $H$, which consists of a number of interaction terms, of which the following four terms are of interest:

$$
H=H_{\mathrm{Z}}+H_{\mathrm{CSA}}+H_{\mathrm{Q}}+H_{\mathrm{D}}
$$

where $H_{Z}$ is the Zeeman term and the next three terms represent the static interactions. The static interactions have a common scaling term, $(1 / 2)\left(3 \cos ^{2} \theta-1\right)$, which is the second Legendre polynomial, $P_{2}(\theta)$, where $\theta$ is an angle that relates the principle coordinate system of the specific interaction to the main magnetic field $\left(B_{0}\right)$ [12]. This will have the following effects on the observed NMR spectrum:

- $H_{\mathrm{CSA}}$ - The chemical shift anisotropy that represents the effect of induced magnetic fields due to orbital electronic motions, i.e. the chemical shift. Will change the chemical shift of the spectrum with a factor proportional to $P_{2}(\theta)$.

- $H_{\mathrm{Q}}$ - The quadrupole interaction between the nuclear quadrupole moment and the surrounding electric field gradient. Will scale the quadrupolar coupling constant observed for nuclei with spin $>$ $1 / 2$ according to $P_{2}(\theta)$.

- $H_{\mathrm{D}}$ - The dipolar interaction that represents the magnetic interaction between dipoles. Due to simultaneous coupling to several different dipoles this term will give rise to a linebroadening, which is proportional to $P_{2}(\theta)$.

For $\cos \theta=1 / \sqrt{3}$, i.e. $\theta=54.7^{\circ}$, the scaling term $(1 / 2)\left(3 \cos ^{2} \theta-1\right)$ becomes zero and the static interactions "magically" disappear. This condition is generally hard to obtain since $\theta$ is distributed randomly in a non-oriented sample so one has to use special techniques in order to remove the static interactions. In the commonly used solid-state NMR technique, where magic angle spinning (MAS) is utilized, the sample is transferred into a rotor that can be spun at very high spinning rates. The rapid 
spinning of the sample causes all the static interactions to be projected onto the spinning axis of the rotor, which is then turned to the magic angle $\left(54.7^{\circ}\right)$ with respect to $B_{0}$, thereby removing all the static interactions in the sample [16]. For liquid crystalline bilayers, on the other hand, the fast lateral reorientation and intermolecular rotation about the long axis of the molecules takes the role of the rotor and partially averages the interactions to leave only the part that is parallel to the normal with the bilayer. Thus, the interactions are all scaled by $P_{2}\left(\theta_{\mathrm{LD}}\right)$, where $\theta_{\mathrm{LD}}$ is the angle between the main magnetic field and the bilayer normal, and if the sample can be macrocopically oriented with the bilayers aligned with the normals in the same direction, the sample can be adjusted into $\theta_{\mathrm{LD}}=54.7^{\circ}$. Generally, the ${ }^{1} \mathrm{H}$ NMR spectra for such samples show linewidths that are reduced from several $\mathrm{kHz}$ (unoriented) to the order of hundreds of $\mathrm{Hz}$ or less (oriented) [20]. Besides the possibility of obtaining resolved spectra for anisotropic systems, oriented samples can also be used to study orientation dependent phenomena, such as spin relaxation.

Figure 1 illustrates the effect of the quadrupole interaction on the deuterium spectrum of an oriented lipid bilayer sample. Similar illustrations on the CSA and dipolar interactions are shown in a recently published review [20].

\section{Preparation of macroscopically aligned lipid bilayers}

A number of methods has been employed to obtain macroscopically oriented lipid bilayers. The methods can roughly be divided according to weather a single bilayer or a stack of several bilayers is required. In the former case Langmuir-Blodgett films [3] and vesicle adsorption onto a solid substrate [4] can be used. In the latter case, molecules forming structures with an anisotropic diamagnetic susceptibility might orient spontaneously if the viscosity is small enough [24,29]. If this does not apply, the most commonly used method is spontaneous orientation of a thin film of lipids on a solid substate, e.g. glass plates or mica. This method has a history back in the early 70's when the first pfg-NMR diffusion measurements were performed $[2,14]$. In general there is no "best" method of obtaining a good oriented sample and experience combined with a flexible thinking has produced many different approaches to this problem $[9,11,15,18,19]$.

In our laboratory good results have been obtained by the following procedure. Lipids dissolved in a 1:4 mixture of methanol : 1-propanol are deposited onto thoroughly cleaned, but otherwise untreated, glass plates to a concentration of about $5-15 \mu \mathrm{g} / \mathrm{mm}^{2}$. The solvent is evaporated and the plates are placed into high vacuum for at least 4 hours to remove traces of solvent. The choice of solvent mixture gives a good adhesion to the glass surface and results in thin films covering the glass plates. The choice of solvent can be critical [19] and in some cases the glass surface has to be modified in order to obtain a suitable degree of hydrophilicity for the lipids to adhere to it. The plates are then stacked on top of each other and placed into a glass tube with square cross section. Typically, 35 (5 mm tube) or 60 (10 mm tube) plates are used for one sample.

The sample tube is placed for several days in a humid atmosphere above the gel to $\mathrm{L}_{\alpha}$ phase transition temperature. During this time hydrated and oriented bilayers are formed. Hydration by humid atmosphere is generally preferred over the use of liquid water since addition of liquid water disrupts the bilayers and results in the formation of vesicular structures. This occurs especially when more than one glass plate is needed for obtaining a sufficient signal/noise ratio. If the glass plates are stacked before hydration, addition of liquid water will disrupt the bilayers as water is sucked in between the plates by capillary forces. Attempts to stack prehydrated plates often results in mechanical disruption of the lipid bilayers. 

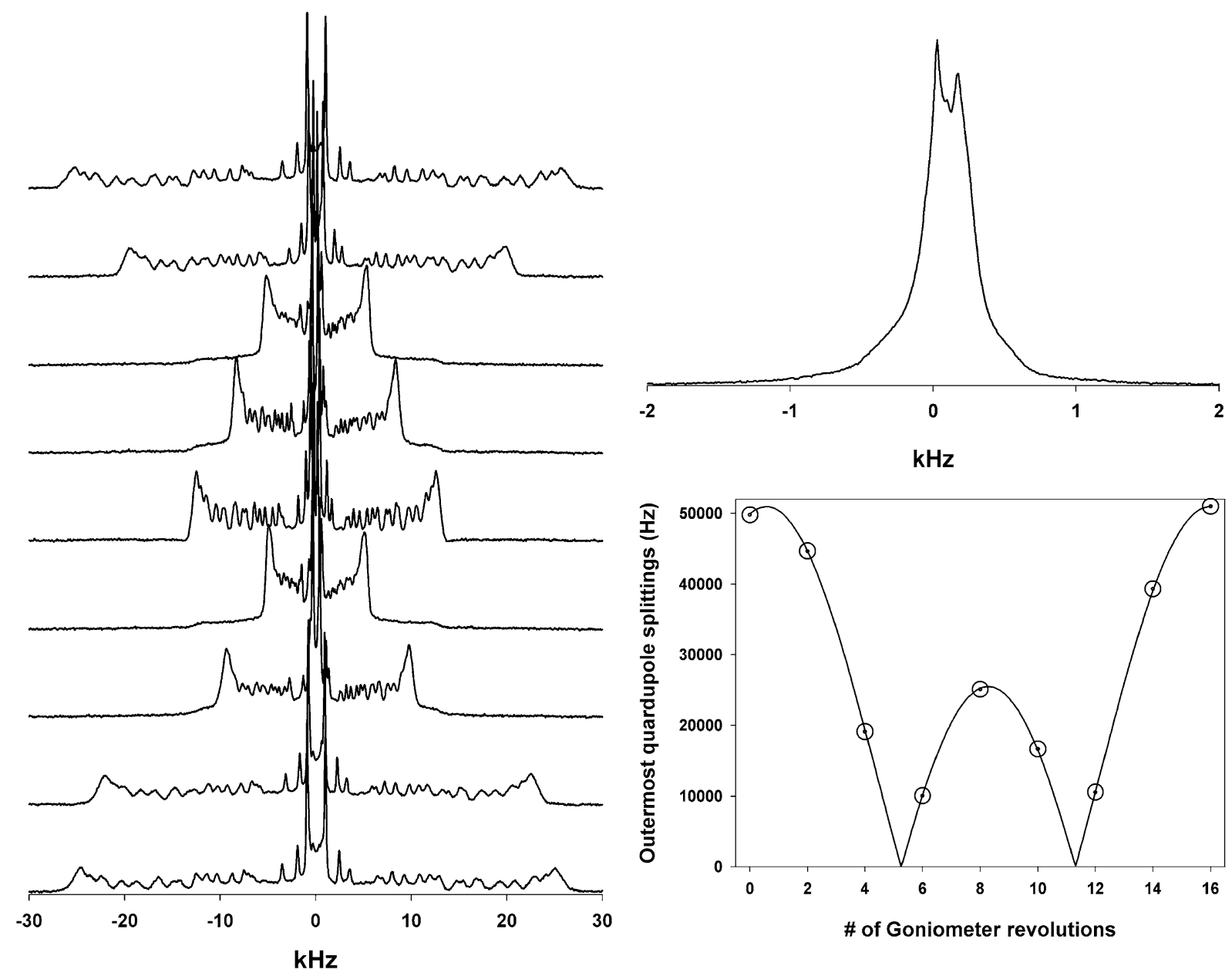

Fig. 1. This figure shows the effects of the quadrupole interaction on the ${ }^{2} \mathrm{H}$ spectra of an oriented sample of a hydrated lipid bilayer composed of equimolar amounts of dioleoylphosphatidylcholine (DOPC) and perdeuterated dipalmitoylphosphatidylcholine (DPPC) at $50^{\circ} \mathrm{C}$. The spectrum consists of signals from all deuterons of the perdeuterated lipid and, due to the macroscopic orientation, is a sum of pairs of peaks with a frequency separation, $\Delta \nu_{q}$, governed by the averaged quadrupole coupling constant, $\Delta \nu_{q}^{0}$, and the orientation

$$
\Delta \nu_{q}=\Delta \nu_{q}^{0} P_{2}\left(\theta_{\mathrm{LD}}\right)
$$

in which $\Delta \nu_{q}^{0}$ is determined by the so-called order parameter of the C-D bond [12]. Due to the different order parameter of the different deuterons the spectrum will be split into a large number of peaks, all sharing the same value of $P_{2}\left(\theta_{\mathrm{LD}}\right)$ but with differing $\Delta \nu_{q}^{0}$. In the left figure the sample was oriented with $\theta_{\mathrm{LD}}$ close to zero degrees, and the sample was then rotated by two revolutions of the goniometer screw for each subsequent spectrum. This results in the splitting of the peaks to change according to $P_{2}\left(\theta_{\mathrm{LD}}\right)$. The figure on the bottom right plots the outermost splittings against the number of revolutions of the goniometer. The fit gives a maximum splitting, $\Delta \nu_{q}^{0}$, of $50.9 \mathrm{kHz}$, corresponding to an order parameter of 0.20 , and $11.8^{\circ}$ of sample rotation/turn of the goniometer. The top right figure shows the spectrum at the magic angle $\left(\theta_{\mathrm{LD}}=54.7^{\circ}\right)$, where all the splittings have coalesced into the isotropic spectrum in which the two main peaks come from the choline headgroup and the acyl chain deuterons, respectively. This difference in isotropic chemical shift gives rise to the slight asymmetry in the innermost splittings seen in the left figure. 
Finally, after obtaining the desired water content (checked by weighing), the tube is sealed and the sample is left another day or two for final equilibration. This procedure results in that when the sample is watched between crossed polarizers along the bilayer normal, large dark areas are observed, since the sample is optically isotropic along the bilayer normal [20]. Mostly more than $85 \%$ of the sample is found to be oriented as determined from ${ }^{31} \mathrm{P}$ NMR, in which the area under the signal from the oriented part of the sample is compared to the total area [20].

\section{The pfg-NMR method for measuring lipid translational diffusion}

The NMR methods with pulsed magnetic field gradients provide some of the most attractive techniques for studies of molecular transport and the applicability of the NMR diffusion techniques has been growing fast due to many improvements of the NMR equipments for diffusion and microscopy [5]. One of the most successful applications of pfg-NMR is its use in extracting structural information about heterogeneous systems such as complex liquids and liquid crystals $[13,28,31]$ and in special, the lipid lateral diffusion coefficients in a macroscopically oriented $\mathrm{L}_{\alpha}$ phase can be directly measured $[13,14$, 20].

The use of NMR for diffusion measurements rests on the ability to create transverse magnetization with a presession rate that is dependent on the local magnetic field. The details of this method are beyond the scope of this article and the interested reader is referred to previously published reviews [13,28,31]. Here, only a brief introduction will be given.

Spins moving in an inhomogenous magnetic field will experience a varying precession rate and the refocussing of the magnetization will in general not be completed after the application of a refocussing pulse. This effect is enhanced by the application of magnetic field gradients during the dephasing/rephasing periods and the resulting attenuation of the echo amplitude [30]

$$
A=\sum_{i} A_{0 i} \exp \left[-\gamma^{2} g^{2} \delta^{2} D_{i}(\Delta-\delta / 3)\right]
$$

is utilized to obtain the self-diffusion coefficient, $D$, of the molecules. In this equation the summation goes over all diffusion components. $A_{0 i}$ is the echo amplitudes without applied gradients, $\gamma$ is the gyromagnetic ratio, $\Delta$ is the time interval between gradient pulses and $\delta$ and $g$ are the duration and amplitude of the pulsed field gradients, respectively. The initial echo amplitude $A_{0 i}$ is determined by the longitudinal and transverse NMR relaxation times. Usually the second half of the spin echo is collected and Fourier transformed to obtain the spectrum in the frequency domain. In this case the amplitude at each frequency channel is governed by Eq. (2). In order to separate the bandshapes corresponding to differently diffusing species the use of the CORE method for global analysis of the entire data set has proven useful [32]. The CORE analysis provides values of the diffusion coefficients together with the individual amplitudes of the diffusing components for each frequency channel, i.e. the individual bandshapes of the diffusional components are obtained. By applying the analysis to an increasing number of components one can normally decide on the actual number of components from the improvement in the normalized global error square sum. An example of the CORE analysis is presented in Fig. 2.

The most important factor to keep in mind when applying this technique to lipid bilayers is that the inhomogenous broadening of the dipole coupling gives a very fast spin relaxation and in order for the NMR method to be of practical use these interactions must be eliminated by the methods described earlier. To accomplish this a specially built goniometer probe is used (Fig. 3). By turning a rod connected 

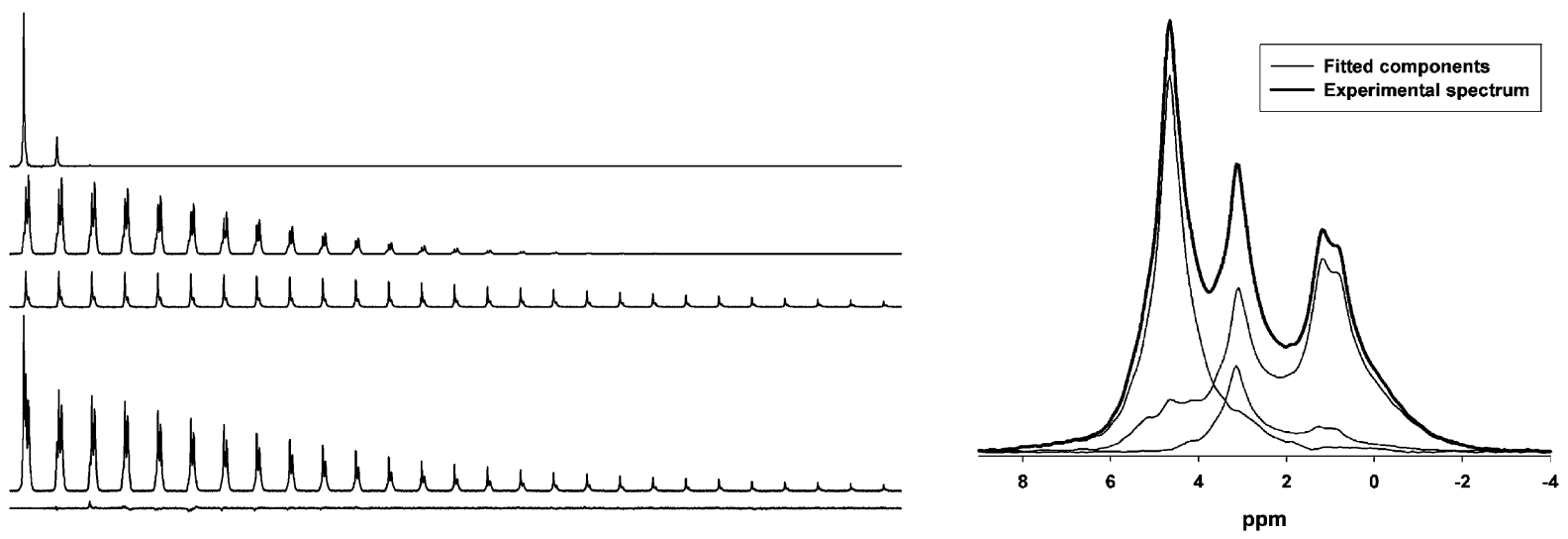

Fig. 2. An example of the data analysis using CORE in a raft-forming sample at $21^{\circ} \mathrm{C}$. The sample is composed of hydrated bilayers of DOPC/DPPC/cholesterol (35/35/30 mol\%). The left figure shows stacked plots of 27 spectra with the gradient strength increasing from 0.238 to $9.52 \mathrm{~T} / \mathrm{m}$ in equal steps. Shown from top to bottom is the fast component of water $\left(D=132 \mu \mathrm{m}^{2} / \mathrm{s}\right)$, the fast lipid component $\left(D=2.67 \mu \mathrm{m}^{2} / \mathrm{s}\right)$, the slow lipid component $\left(D=0.47 \mu \mathrm{m}^{2} / \mathrm{s}\right)$, the experimental spectra, and the difference between the experiment and the fit (the residuals). The figure to the right shows the experimental spectrum at $0.238 \mathrm{~T} / \mathrm{m}$ strength (bold line), together with the decomposition into three spectra, each with a separate diffusion coefficient, obtained from the CORE analysis. The dominant peaks for the lipids originate from the choline headgroup (3.1 ppm) and the chain methylene $(1.1 \mathrm{ppm})$ and methyl $(0.8 \mathrm{ppm})$. Note the difference in the lipid lineshapes for the two components, caused by different relaxation rates in the two phases.

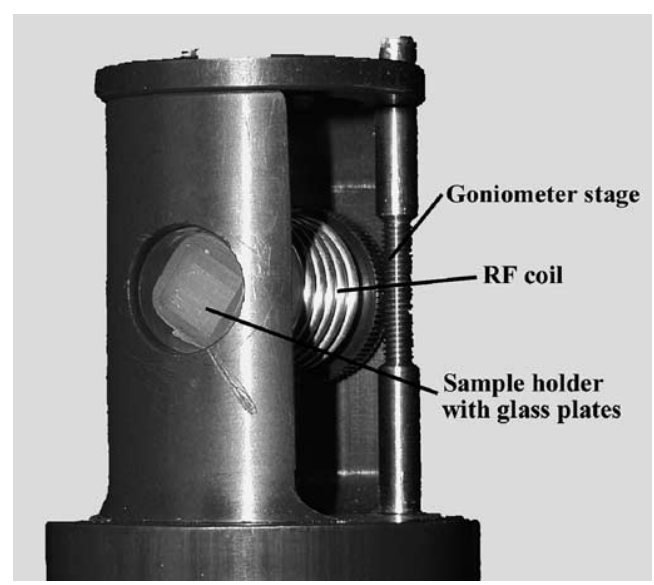

Fig. 3. A picture of the goniometer stage of the NMR probe. The sample holder (10 mm OD) with a square glass tube holding the oriented sample is seen on the left. (The sealing of the square tube is removed to show the glass plates.) On the right hand side, the angular gear that allows rotation of the sample holder around its long axis is seen.

to the goniometer stage the sample can be rotated from outside of the magnet so that the bilayer normal makes an angle of $54.7^{\circ}$ with respect to $B_{0}$, thereby canceling the dipole interaction and producing an isotropic-like spectrum. In doing this the effective transverse relaxation time changes from sub-ms to 20-50 ms, thereby enabling the spin-echo experiment to be performed.

In the NMR experiment the translational diffusion is measured in the direction of the magnetic field gradient, which normally is directed parallel with $B_{0}$. For a lipid membrane the observed diffusion co- 
efficient, $D$, depends on the orientation and two diffusion coefficients, $D_{\mathrm{L}}$ and $D_{\perp}$. $D_{\mathrm{L}}$ is the lateral diffusion coefficient for motion parallel with the membrane plane, and $D_{\perp}$ stands for the motion perpendicular to the bilayer. Then,

$$
D=D_{\mathrm{L}} \sin ^{2} \theta_{\mathrm{LD}}+D_{\perp} \cos ^{2} \theta_{\mathrm{LD}}
$$

For a bilayer oriented at the magic angle $\sin ^{2} \theta_{\mathrm{LD}}=2 / 3$ and, since it is reasonable to assume that $D_{\perp}$ is orders of magnitude smaller than $D_{\mathrm{L}}$ so that the second term in Eq. (3) can be neglected, $D_{\mathrm{L}}$ is given by $D_{\mathrm{L}}=1.5 D$.

Our lab is equipped with a $10 \mathrm{~mm}{ }^{1} \mathrm{H}$ probe for a $100 \mathrm{MHz}$ NMR spectrometer with a maximum gradient strength of $3 \mathrm{~T} / \mathrm{m}$ (routinely used for lipid diffusion measurements in the order of $1-10 \mu \mathrm{m}^{2} / \mathrm{s}$ ) and with a $5 \mathrm{~mm}$ dual ${ }^{1} \mathrm{H} / \mathrm{X}$ probe for a $400 \mathrm{MHz}$ system that is capable of giving gradient strengths up to $10 \mathrm{~T} / \mathrm{m}$ (for slow diffusion of the order of $0.01-0.1 \mu \mathrm{m}^{2} / \mathrm{s}$ and diffusion of isotopically enriched molecules, e.g. with ${ }^{2} \mathrm{H},{ }^{31} \mathrm{P},{ }^{19} \mathrm{~F}$ and ${ }^{13} \mathrm{C}[22,23]$. For measurements in which the gradient direction can be varied with respect to $B_{0}$, we use a microimaging system with a goniometer sample holder [34].

\section{Applications}

\subsection{Domains and rafts in lipid bilayers}

During the past decade the organization of lateral structures in biological membranes and their importance for biological activity have received increasing attention as the importance of domain formation and percolation phenomena to cellular processes have been recognized [1,25-27,33]. We have witnessed an emergence of interest in a specific type of cholesterol rich domains, often referred to as membrane rafts [17,27], and since the raft-concept was coined in 1997 [26], the number of annual publications on rafts has steadily increased.

Since the translational motion of lipids will be affected by the presence of microdomains in the lipid bilayer, it is possible to use the pgf-NMR diffusion method to investigate such domains [6-8, 20-23]. The crucial parameters determining the diffusional behaviour is the exchange times, the partitioning coefficients, and the diffusion coefficients for lipid species in the different domains [10]. Figure 4 shows an example of the different dependence on cholesterol concentration of the lipid diffusion coefficient in systems forming homogeneous bilayers, microdomain rafts, and rafts of macroscopic sizes.

With this technique we have successfully investigated several potentially raft-forming systems in order to get an understanding of the physico-chemical mechanisms in the raft-forming process. Recently, we have extended the method to isotopically labelled molecules, which allows us to measure the diffusion of each individual lipid species in the raft mixture [22]. In order to gain further insight we are presently investigating sterols involved in the biosynthesis of cholesterol as well as sterols that perform the same function as cholesterol in plants.

Lipid diffusion can also be analyzed in the context of solid, inpermeable obstacles, such as integral proteins and gel patches. In such systems the diffusion coefficient will be dependent on the total fraction of obstacles as well as the size and shape of the obstacles [25]. In a combined differential scanning calorimetry and pfg-NMR study we have investigated the effect of the incorporation of gramicidin $D$ (a transmembrane peptide) and gel patches on the diffusion of dimyristoylphosphatidylcholine in oriented bilayers. We were able to draw conclusions both of the number of lipids associated with the peptide and the shape of the obstacles [21]. 


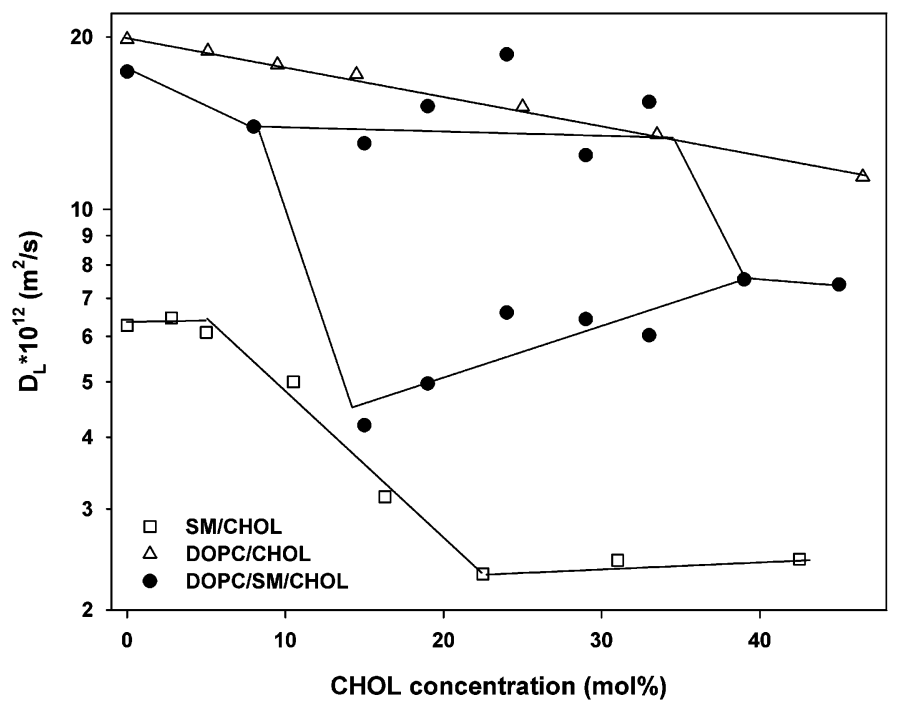

Fig. 4. Lipid lateral diffusion in cholesterol-containing bilayers at $50^{\circ} \mathrm{C}$. This figure shows the different behaviour obtained for three different bilayer component organizations. Triangles: DOPC/cholesterol. This system forms a microscopically homogeneous system and the lipid diffusion varies continuously as the cholesterol concentration increases [6]. Squares: egg sphingomyelin (eSM)/cholesterol. This system exhibits domain formation in the range of 6-22 mol\% cholesterol. Due to fast exchange the observed diffusion coefficient will be a weighted average of the diffusion coefficients in the separate phases. This is an indication that the domains are smaller than the mean diffusion length (ca $1 \mu \mathrm{m})$. In the one phase regions on each side of the two-phase area the diffusion coefficients are almost constant [6]. Circles: DOPC/eSM/cholesterol. Macroscopic phase separation occurs in this system between 10 and $37 \mathrm{~mol} \%$ cholesterol. Due to the large domains formed the lipids will mainly move within the same domain during the measurement time (50-200 ms) and exchange between the phases will therefore be slow on this timescale. This results in separately observed diffusion coefficients corresponding to diffusion in the two phases [7]. A fourth case, not illustrated, is also possible in which the exchange time between the domains is comparable to the diffusion time. In this case two-component diffusion will also be observed but then both the pre-exponential factors and the apparent diffusion coefficients will be functions of relaxation times, diffusion coefficients and lifetimes of the lipids in the two phases [10].

\subsection{Anisotropic diffusion in oriented systems}

In the study of lipid diffusion the motion parallel to the bilayer normal is orders of magnitudes smaller than that within the bilayer plane. This is not the case for molecules that are partially dissolvable in both water and oil, since this feature allows the molecules to pass through both the water layers and the oily hydrocarbon core of the stacked bilayers. In this case the diffusion along the normal will be slowed down as compared to that along the layers and this anisotropy in the diffusion can be measured with pfg-NMR.

For this application we use the ability of the imaging equipment on the $400 \mathrm{MHz}$ spectrometer to produce pulsed field gradients in any direction, thereby allowing for diffusion measurements at any angle to the bilayer normal. With this method we have investigated water diffusion anisotropy in bilayers and found that the water diffuses at least two orders of magnitude slower along the normal than perpendicular to it [34]. Further studies are planned in order to utilize this method in studies of membrane permeability and controlled drug release.

\section{Acknowledgement}

This work was supported by the Swedish Research Council. 


\section{References}

[1] R.G.W. Andersson, Annu. Rev. Biochem. 67 (1998), 199.

[2] S.A. Asher and P.S. Pershan, Biophys. J. 27 (1979), 393.

[3] K.B. Blodgett, J. Am. Chem. Soc. 57 (1935), 1007.

[4] A.A. Brian and H.M. McConnell, Proc. Natl. Acad. Sci. 81 (1984), 6159.

[5] P.T. Callaghan, Principles of Nuclear Magnetic Resonance Microscopy, Clarendon Press, Oxford, 1991.

[6] A. Filippov, G. Orädd and G. Lindblom, Biophys. J. 84 (2003), 3079.

[7] A. Filippov, G. Orädd and G. Lindblom, Biophys. J. 86 (2003), 891.

[8] A. Filippov, G. Orädd and G. Lindblom, Langmuir 19 (2003), 6397.

[9] K.J. Hallock, K. Henzler Wildman, D.-K. Lee and A. Ramamoorthy, Biophys. J. 82 (2002), 2499.

[10] J. Kärger, H. Pfeifer and W. Heink, in: Advances in Magnetic and Optical Resonance, W.S. Warren, ed., Academic Press, Inc., San Diego, CA, 1988, p. 1

[11] J. Katsaras, Biophys. J. 73 (1997), 2924.

[12] G. Lindblom, in: Advances in Lipid Methodology, W.W. Christie, ed., The Oily Press Ltd., Dundee, 1996, p. 133.

[13] G. Lindblom and G. Orädd, Prog. NMR Spectrosc. 26 (1994), 483.

[14] G. Lindblom and H. Wennerström, Biophys. Chem. 6 (1977), 167.

[15] F.M. Marassi and K.J. Crowell, J. Magn. Res. 161 (2003), 64.

[16] M.M. Maricq, J. Chem. Phys. 70 (1979), 3300.

[17] H.M. McConnell and M. Vrljic, Annu. Rev. Biophys. Biomol. Struct. 32 (2003), 469.

[18] U. Mennicke and T. Salditt, Langmuir 18 (2002), 8172.

[19] F. Moll III and T.A. Cross, Biophys. J. 57 (1990), 351.

[20] G. Orädd and G. Lindblom, Magn. Res. Chem. 42 (2004), 123.

[21] G. Orädd and G. Lindblom, Biophys. J. 87 (2004), 980.

[22] G. Orädd and G. Lindblom, Biophys. J. (2005), In press.

[23] G. Orädd, G. Lindblom and P.W. Westerman, Biophys. J. 83 (2002), 2702.

[24] C.R. Sanders, B.J. Hare, K.P. Howard and J.H. Prestegard, Prog. NMR Spectrosc. 26 (1994), 421.

[25] M.J. Saxton, Curr. Top. Membr. 48 (1999), 229.

[26] K. Simons and E. Ikonen, Nature 387 (1997), 569.

[27] K. Simons and W.L. Vaz, Annu. Rev. Biophys. Biomol. Struct. 33 (2004), 269.

[28] O. Söderman and P. Stilbs, Prog. NMR Spectrosc. 26 (1994), 445.

[29] R. Soong and P.M. Macdonald, Biophys. J. 88 (2005), 255.

[30] E.O. Stejskal and J.E. Tanner, J. Chem. Phys. 42 (1965), 288.

[31] P. Stilbs, Prog. NMR Spectrosc. 19 (1987), 1.

[32] P. Stilbs, K. Paulsen and P.C. Griffiths, J. Phys. Chem. 100 (1996), 8180.

[33] W.L. Vaz and P.F.F. Almeida, Curr. Opin. Struct. Biol. 3 (1993), 482.

[34] P. Wästerby, G. Orädd and G. Lindblom, J. Magn. Res. 157 (2002), 156. 


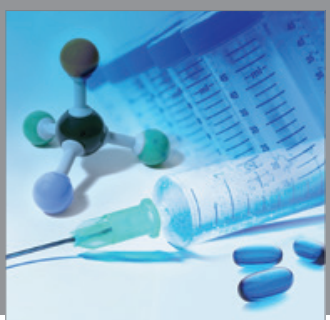

International Journal of

Medicinal Chemistry

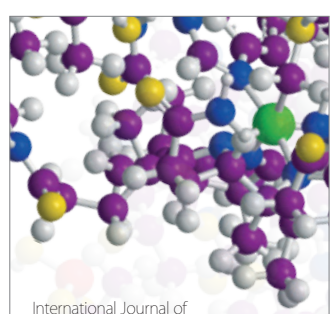

Carbohydrate Chemistry

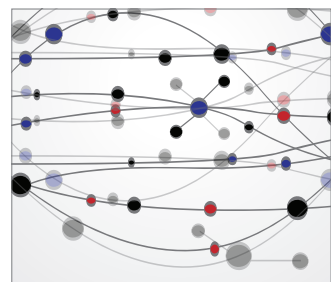

The Scientific World Journal
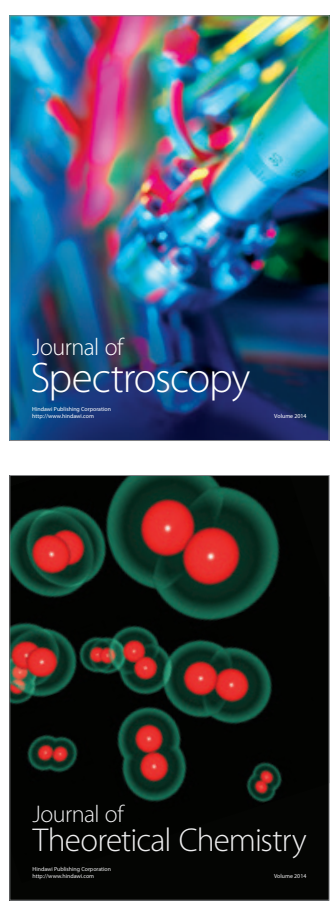
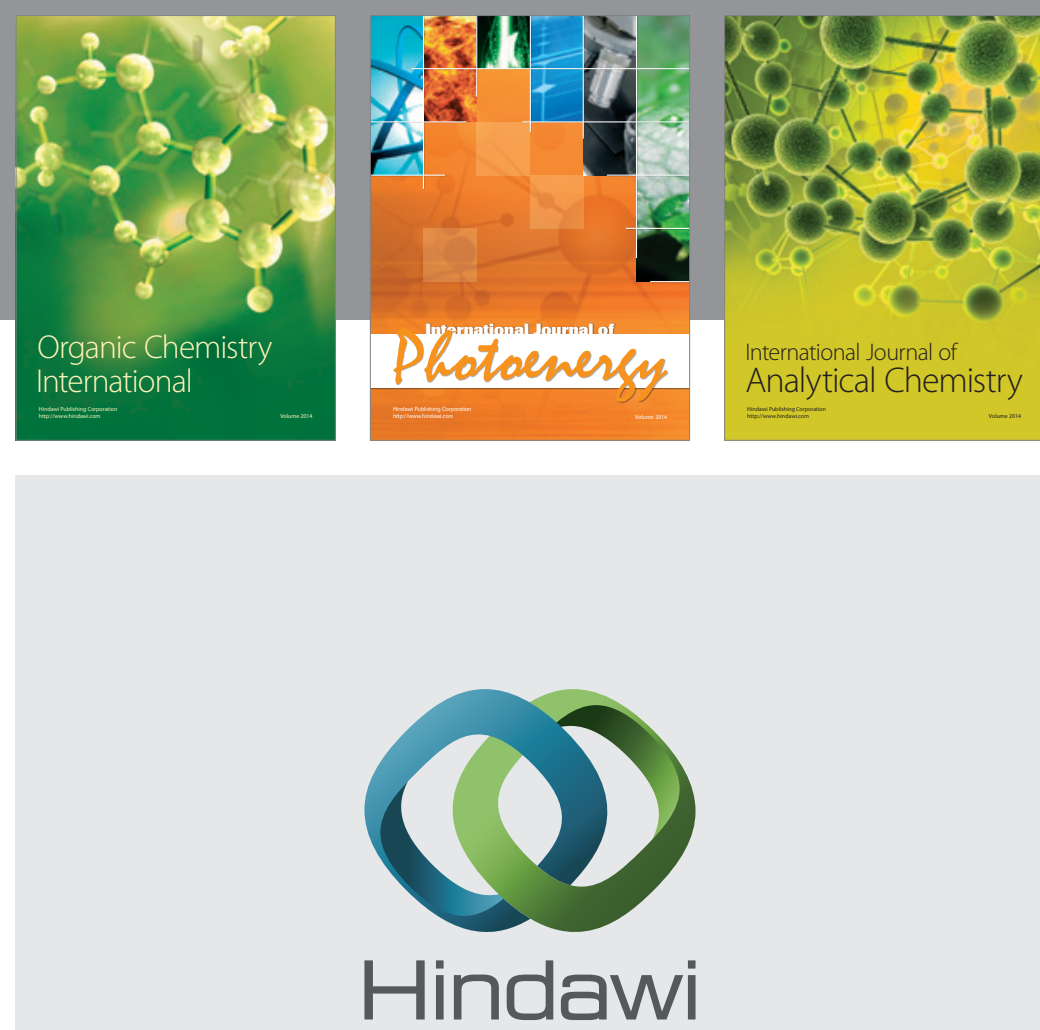

Submit your manuscripts at

http://www.hindawi.com
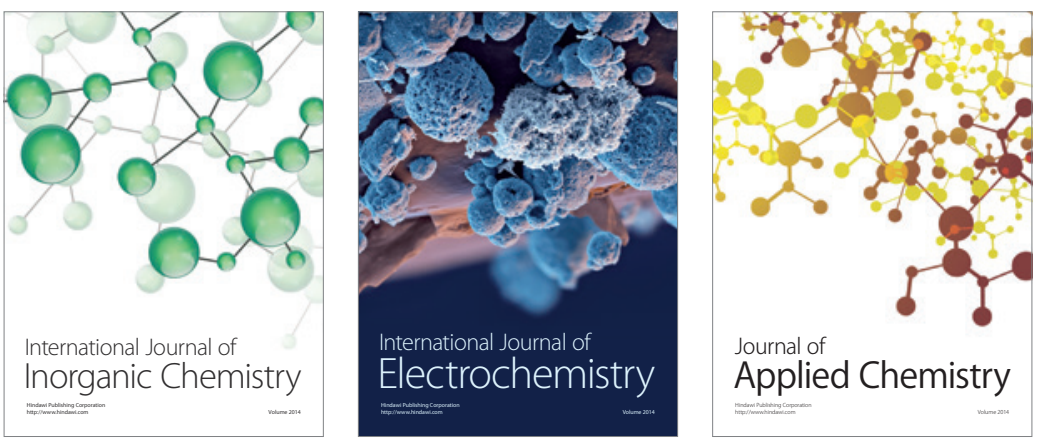

Journal of

Applied Chemistry
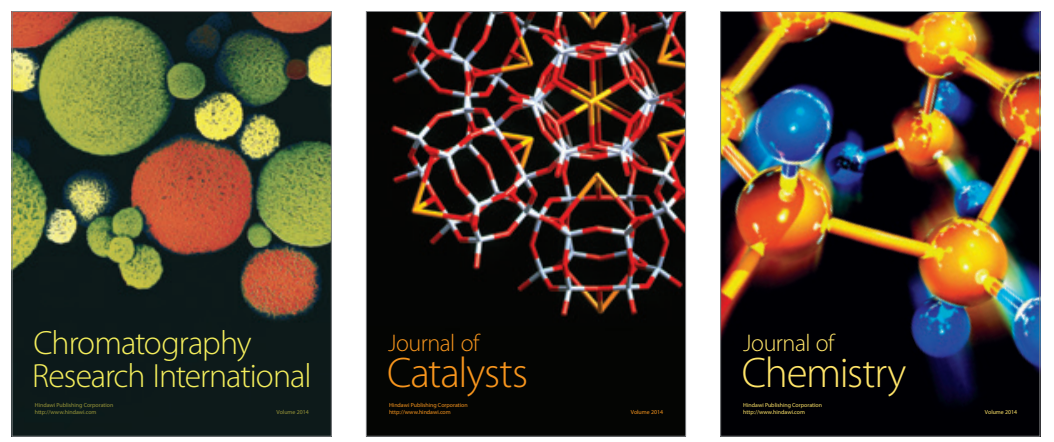
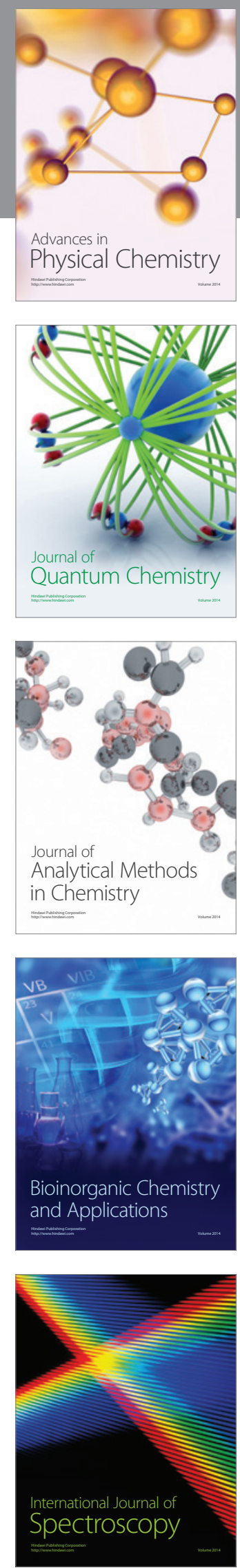